\title{
The antimicrobial susceptibility, biofilm formation and genotypic profiles of Staphylococcus haemolyticus from bloodstream infections
}

\author{
Patricia Vollú Silva ${ }^{1,3}+{ }^{+}$, Raquel Souza Cruz², Luiz Sérgio Keim¹, Geraldo Renato de Paula ${ }^{3}$, \\ Bernadete Teixeira Ferreira Carvalho², Leonardo Rocchetto Coelho ${ }^{4}$, Maria Cícera da Silva Carvalho ${ }^{2}$, \\ Joel Mauricio Corrêa da Rosa ${ }^{5}$, Agnes Marie Sá Figueiredo², Lenise Arneiro Teixeira ${ }^{1,3}$ \\ ${ }^{1}$ Faculdade de Medicina ${ }^{3}$ Faculdade de Farmácia ${ }^{5}$ Instituto de Matemática e Estatística, Universidade Federal Fluminense, \\ Niterói, RJ, Brasil 'Instituto de Microbiologia Paulo de Góes, Universidade Federal do Rio de Janeiro, Rio de Janeiro \\ ${ }^{4}$ Departamento de Ensino de Graduação, Universidade Federal do Rio de Janeiro, Macaé, RJ, Brasil
}

We analysed the antimicrobial susceptibility, biofilm formation and genotypic profiles of 27 isolates of Staphylococcus haemolyticus obtained from the blood of 19 patients admitted to a hospital in Rio de Janeiro, Brazil. Our analysis revealed a clinical significance of $36.8 \%$ and a multi-resistance rate of $92.6 \%$ among these isolates. All but one isolate carried the mecA gene. The staphylococcal cassette chromosome mec type I was the most prevalent mec element detected (67\%). Nevertheless, the isolates showed clonal diversity based on pulsed-field gel electrophoresis analysis. The ability to form biofilms was detected in $66 \%$ of the isolates studied. Surprisingly, no icaAD genes were found among the biofilm-producing isolates.

Key words: Staphylococcus haemolyticus - SCCmec - biofilm

Staphylococcus haemolyticus isolates are saprophytic bacteria with the ability to colonise human skin and mucosal membranes. However, these coagulasenegative bacteria have recently emerged as hospitalassociated pathogens, mainly in immune-compromised patients. Recent studies have identified $S$. haemolyticus as an important cause of primary bloodstream infections associated with the use of central venous catheters (CVC) (Keim et al. 2011, Barros et al. 2012, Bouchami et al. 2012). Notably, S. haemolyticus isolates exhibit high resistance profiles and quite frequently display reduced susceptibility to glycopeptide antibiotics (Ahlstrand et al. 2011, Barros et al. 2012). The bacterial ability to form biofilms is thought to be an important mechanism involved in CVC-related infections and in other deviceassociated diseases. Biofilm formation among staphylococci is mediated by the ica operon, which encodes the enzymes involved in the synthesis of poly-N-acetylglucosamine/polysaccharide intercellular adhesin (PNAG/ PIA). Nevertheless, PNAG/PIA-independent biofilm matrices have been described in these cocci, including Staphylococcus epidermidis (Araujo et al. 2006). However, the biofilms formed by S. haemolyticus have not yet been subjected to more detailed studies.

In this paper, 27 consecutive isolates initially identified as methicillin-resistant $S$. haemolyticus (MRSHa)

\footnotetext{
doi: 10.1590/0074-0276108062013022

Financial support: FAPERJ, CNPq, PROPPi-UFF, European Commission's FP7/Marie Curie International Research Staff Exchange Scheme NANO_GUARD (PIRSES-GA-2010-269138)

+ Corresponding author: patyvollu@ig.com.br

Received 21 December 2012

Accepted 25 June 2013
}

using the Vitek Automatic System with GPS-105 [BioMérieux Brasil, Rio de Janeiro (RJ), Brazil] were studied. These isolates were obtained from blood samples collected from 17 adult and two paediatric patients who were admitted in different clinical settings to a tertiary hospital in RJ from 2007-2008. Because different isolates recovered from the blood of an individual patient can display different genetic profiles, more than one isolate per patient was included in this study. The exclusion criterion was the presence of isolates from the same patient displaying an identical pulsed-field electrophoresis pattern. In such cases, only one of the repeat isolates was included. The identification of the isolates was confirmed via polymerase chain reaction (PCR) amplification of the mvaA gene, as described previously (Barros et al. 2012). $S$. haemolyticus isolates were considered clinically significant based on the criteria described by the Centers for Diseases Control and Prevention (CDC), which includes: (i) a patient with a recognised pathogen cultured from two or more blood cultures collected within a period of two days, (ii) risk factors for bloodstream infection, (iii) classical clinical manifestations and (iv) associated clinical laboratory findings (Horan et al. 2008).

Antibiogram tests were carried out as recommended by the Clinical and Laboratory Standards Institute (CLSI 2011). The minimum inhibitory concentration (MIC) was determined for vancomycin and teicoplanin (TEI) (CLSI 2011). Multi-resistance was defined as resistance to three or more classes of antimicrobials, including $\beta$-lactams. High-level multi-resistance was defined as an isolate displaying resistance to six or more drugs. PCR was used to detect mecA (Araujo et al. 2006), encoding methicillin resistance and to determine the type of staphylococcal cassette chromosome mec (SCCmec) (Boye et al. 2007, Berglund et al. 2008, Zhang et al. 2009). PCR analysis was also employed to assess the ica operon (Araujo et al. 2006). The icaAD primers exhibited $100 \%$ 
correspondence to the ica sequence of $S$. haemolyticus (GenBank accession FJ472951.2). Additionally, a PCR probe amplified with specific $i c a A D$ primers (Araujo et al. 2006) was labelled using the DIG High Prime DNA Labelling and Detection Starter Kit II (Roche Diagnostics, Mannhein, Germany) and was used for dot-blot hybridisation experiments with chromosomal DNA to confirm the PCR-negative results for icaAD genes. The ica-positive S. epidermidis isolate 70D (Araujo et al. 2006) was used as a positive control for the assessment of ica via PCR or dot-blot-hybridisation and as a negative control for the experiment involving proteinase $\mathrm{K}$. Biofilm formation was investigated using a microtitrebased system and the biofilm units (BU) was calculated as previously defined (Araujo et al. 2006).

The 27 isolates were analysed through pulsed-field gel electrophoresis (PFGE) following chromosomal DNA digestion with the SmaI endonuclease (Teixeira et al. 1995). PFGE types were defined on the basis of DNA-banding patterns according to visual criteria. Isolates exhibiting PFGE patterns that differed from one another by fewer than four bands were assigned a common capital letter, with numerical indices representing subtypes, as described previously (Teixeira et al. 1995). An unpaired Student's $t$ test was used for the majority of statistical analyses, except for the determination of correlations between multi-resistance and biofilms and between high-level resistance among clinically significant and non-significant isolates, for which Fisher's exact probability test was chosen. A level of 0.05 was considered significant for both statistical tests.

Based on the CDC criteria, clinically significant $S$. haemolyticus isolates were collected from seven of the 19 patients studied (36.8\%). Among these individuals, patient 7 (P7) presented two isolates with dissimilar genetic profiles. In this case, the clinically significant $S$. haemolyticus strain could be identified based on the PFGE type of the isolates compared to those excluded from the study due to showing an identical pattern to the significant isolate. In one other patient (P4), the significant isolate could not be identified on the basis of molecular techniques given that the two isolates recovered showed distinct genetic profiles. A total of 13 isolates recovered from eight patients were considered contaminants. Five isolates collected from four patients could not be classified due to a lack of sufficient patient data (Table). Eight isolates were obtained from 19 patients (42.1\%) who presented with malignant tumours, three of whom exhibited community infections (15.8\%), while the remainder showed vascular cerebral accident, alcoholic liver cirrhosis, severe hypertension, type II diabetes mellitus and acute pulmonary oedema. Age data are reported for 14 patients. The mean age ( \pm standard deviation) of adult patients was $50 \pm 22$ years (range, 24-93 years). Additionally, two patients were classified as paediatric ( $>2$ years old).

The gender distribution among the 19 patients tested was close to equal ( $47 \%$ males and $53 \%$ females). Despite the limited number of isolates, $85.7 \%$ (6/7) of the clinically significant patients were female. In contrast, only $25 \%(2 / 8)$ of the contaminated patients were fe- males $(p=0.04)$. We were not able to identify any factor to explain the difference between the two gender groups, except that the two oldest patients were females belonging to the group with clinical significance (Table). Due to the small number of studies on S. haemolyticus epidemiology available in the scientific literature, we could not comparatively analyse the gender data. Thus, studies with a greater number of clinically significant cases are required to clarify this issue.

The great majority of the tested S. haemolyticus isolates displayed multi-resistant profiles $(25 / 27 ; 92.6 \%)$. Among the studied isolates, only two were resistant to all of the drugs tested, except glycopeptides, and these two were therefore defined as high-level resistant isolates (> 8 antimicrobials). These high-level resistant MRSHa isolates were found in the group of clinically significant isolates. Statistical analysis was conducted to compare the high-level resistance detected between clinically significant and non-significant isolates (Table) and the obtained $\mathrm{p}$ value was close to the limit of significance $(\mathrm{p}=0.054)$. It has been suggested that the intensive use of glycopeptides is associated with increasing resistance to this drug, especially among $S$. haemolyticus isolates (Ahlstrand et al. 2011). In the present study, isolate $05 / 08$ displayed TEI resistance ( $\mathrm{MIC}=64 \mu \mathrm{g} / \mathrm{mL}$ ) and was only susceptible to tetracycline, chloramphenicol and rifampicin (Table). During the preparation of this paper, a study was published demonstrating clonal dissemination of linezolidresistant S. haemolyticus isolates exhibiting a G2576T mutation in the 23S rrna gene in a hospital in Brazil (de Almeida et al. 2012). Thus, the high levels of multi-resistance observed in this emergent bacterial pathogen, including resistance to new generation antimicrobial drugs, may have serious implications for the therapeutic treatment of the associated infections. It was suggested that the significant number of IS elements distributed within the $S$. haemolyticus genome may represent hotspots for the acquisition of resistance genes and could promote rearrangement of genomic DNA, which accelerates bacterial diversification (Bouchami et al. 2012).

Only $3.7 \%(1 / 27)$ of the $S$. haemolyticus isolates tested did not carry the mecA gene based on the absence of the expected 164-base pairs (bp) mecA-fragment. However, in DNA obtained from the $m e c A$-negative isolate 220/08, a 415-bp fragment was amplified corresponding to the IS1272 insertion sequence-like element, which is present in some mec islands, including type I, IV and VI (Boye et al. 2007, Berglund et al. 2008, Zhang et al. 2009). Remarkably, isolate $220 / 08$ was resistant to oxacillin (OXA), but was susceptible to cefoxitin in disk tests. In addition, the MIC value recorded for OXA was $1 \mu \mathrm{g} / \mathrm{mL}$. It is well known that the OXA MIC interpretative criteria can lead to overestimation of resistance for certain coagulase-negative staphylococci other than $S$. epidermidis isolates, for which the OXA MICs are between $0.5-2 \mu \mathrm{g} / \mathrm{mL}$ (CLSI 2011).

SCCmec type I was identified among $67 \%$ (18/27) of the tested isolates (Table). This SCCmec type has been described as the most common type found among nosocomial isolates of MRSHa in the south of Brazil (Mom- 


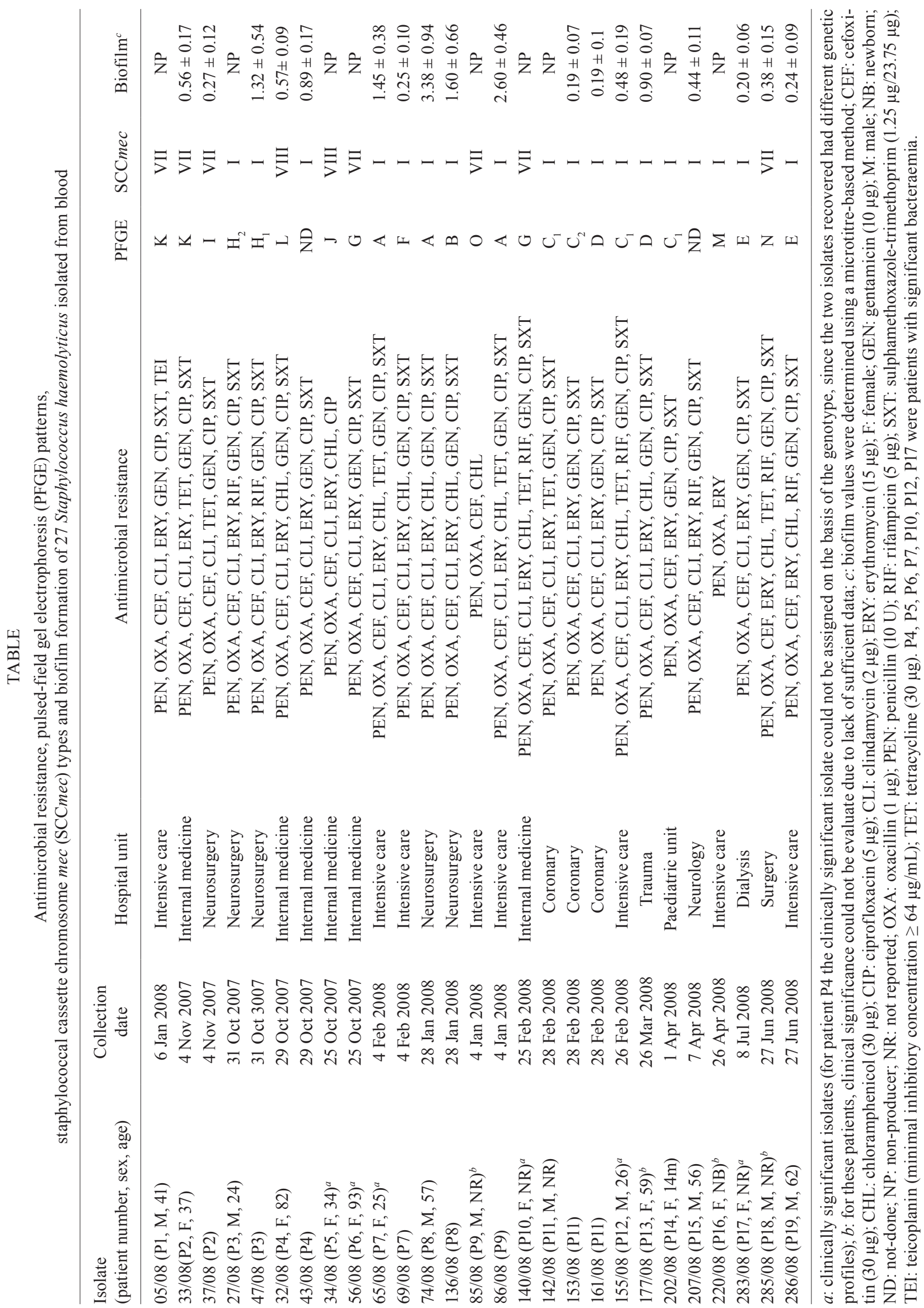


bach et al. 2007). The remaining isolates analysed in the present study were distributed among SCCmec type VII (7/27; 26\%) and type VIII $(2 / 27 ; 7 \%)$ strains. The prevalence of SCCmec V in MRSHa isolates had been reported in previous studies (Barros et al. 2012, Bouchami et al. 2012). However, Zong et al. (2011) found a variety of SCCmec types among 19 MRSHa isolates recovered from a hospital in China. Despite the prevalence of SCCmec I, we observed that these isolates displayed considerable genetic diversity. The 27 analysed isolates were classified into 15 PFGE types and two subtypes (Table). A similar clonal diversity was reported in Brazil for S. haemolyticus isolates collected from another hospital (Barros et al. 2012). In contrast, Bouchami et al. (2012) investigated 36 MRSHa isolates from Tunisia and found that $77 \%$ of the isolates clustered in only four PFGE types. A collection of 72 isolates of $S$. haemolyticus from Norway could also be grouped into only seven different clusters, the largest of which consisted of 16 isolates obtained from neonates treated in a neonatal intensive care unit at the same hospital (Fredheim et al. 2009). Thus, it is possible that this difference may be because the patients included in the present study came from different hospital units (Table), while those studied by the cited authors (Fredheim et al. 2009, Bouchami et al. 2012) came from a more limited number of hospital settings. Similar to what has been observed previously (Bouchami et al. 2012), we found that MRSHa isolates displaying different PFGE types could carry the same SCCmec type (Table), suggesting that horizontal transfer of SCCmec is a relatively common event among MRHSa strains.

Our data showed that 18 of the isolates $(67 \%)$ were able to form biofilms (mean BU: $0.89 \pm 0.86$ ), eight (44\%) of which displayed a high ability to accumulate biofilms (BU $\geq 0.89)$ (Table). Likewise, Fredheim et al. (2009) found that $74 \%$ of the $S$. haemolyticus isolates analysed could produce biofilms. Despite the limited number of isolates tested in this study, 11 contaminants, four clinically significant MRSHa isolates and three other isolates that could not be classified were able to form a biofilm on an inert polystyrene surface (mean BU: $0.91 \pm$ $1.06,0.76 \pm 0.54$ and $1.27 \pm 1.19$ ) (Table). Student's $t$ test showed that there was no significant difference between these populations $(p>0.5)$. Indeed, some contaminants (isolates 47/08, 136/08 and 74/08) showed a high capability to accumulate biofilms (Table). Notably, the only four isolates that were comparatively more susceptible to most of the antimicrobials tested (isolates 34/08, 85/08, $202 / 08$ and 220/08) did not produce biofilms in this study and all of the biofilm producers were classified as showing high-level multi-resistance $(\mathrm{p}<0.01)$ (Table). A relationship between multi-resistance and biofilm formation has also been observed by others (Araujo et al. 2006). Phase variation is a well-known common mechanism of biofilm formation for both ica-positive and negative staphylococci. In this study, we observed that some isolates clustering within a same PFGE type could exhibit quite different biofilm values (Table). It is possible that surface proteins involved in the formation of an ica-negative biofilm matrix might have been influenced by switching transcriptional regulators on or off.
Amplification of the $i c a A D$ genes was not detected in any of the 27 isolates analysed. The absence of the $i c a A D$ genes was confirmed through hybridisation experiments. Similar results have recently been reported by Fredheim et al. (2009), who detected the ica operon in only two of 53 biofilm-producing $S$. haemolyticus isolates. In the present study, the proteinaceous nature of the ica-independent biofilms was indicated by the significant disruption of the mature biofilms generated by the S. haemolyticus isolates following treatment with $6 \mathrm{U} /$ well proteinase $\mathrm{K}$ (Figure). Thus, after treatment with proteinase K, the mean biofilm values for 10 representative MRSHa isolates tested (displaying different biofilm phenotypes) decreased from $0.927 \pm 0.358$ to $0.19 \pm 0.077$ ( $\mathrm{p}=0.002)$. As expected, the ica-dependent biofilm produced by $S$. epidermidis $70 \mathrm{D}(\mathrm{BU}=2.283 \pm 0.204)$ was only slightly affected $(1.648 \pm 0.169 ; \mathrm{p}=0.0408)$ by the proteinase $\mathrm{K}$ treatment (B in Figure). Similar results have been found by others (Fredheim et al. 2009). As expected, treatment of mature biofilms with the carbohydrate-oxidant sodium metaperiodate $(10 \mathrm{mM} /$ well $)$ did not significantly affect pre-formed biofilms $(1.146 \pm 0.824 ; \mathrm{p}=0.26)$. Previous studies have suggested that extracellular DNA is one of the components of the biofilm matrix formed by $S$. haemolyticus (Fredheim et al. 2009). However, the incorporation of DNase I (28U/well) into the microtitre-based system did not significantly impair biofilm formation among these isolates $(0.878 \pm 0.405 ; \mathrm{p}=0.30)$.

In conclusion, our analyses demonstrated that $S$. haemolyticus isolates collected from human blood, representing both clinically significant and contaminant isolates, showed clonal diversity, high-level antimicrobial multi-resistance and an ability to form ica-independent, proteinaceous biofilms. The increasing importance of these multi-resistant isolates in hospital infections in different countries confirms the fitness of these bacteria as opportunistic nosocomial pathogens. Additional studies are necessary to clarify the primary molecules involved in the composition and modulation of the icaindependent biofilms formed by S. haemolyticus.

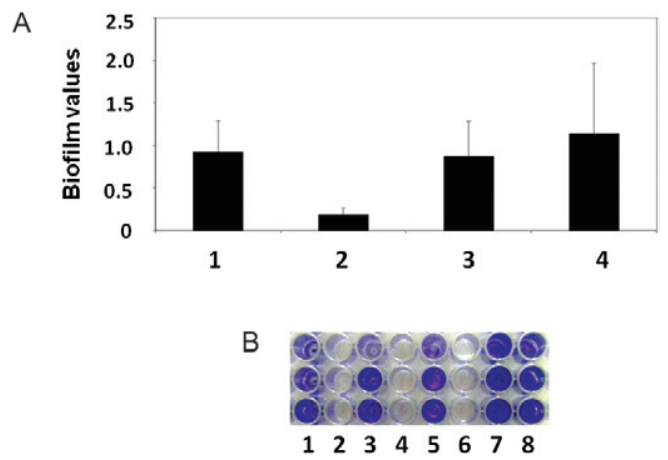

A: biofilms of representative isolates of Staphylococcus haemolyticus (1: untreated; 2: proteinase K; 3: DNase; 4: sodium metaperiodate); B: microtitre based biofilm of representative isolates (1, 2: 33/08; 3 , 4: 37/08; 5, 6: 69/08; 7, 8: ica-dependent biofilm producer Staphylococcus epidermidis 70D); even numbers: proteinase $\mathrm{K}$ treatment; odd numbers: untreated. 


\section{ACKNOWLEDGEMENTS}

To the Microbiology Laboratory, Department of Pathology (HUAP-UFF), for providing the bacterial isolates.

\section{REFERENCES}

Ahlstrand E, Svensson K, Persson L, Tidefelt U, Söderquist B 2011. Glycopeptide resistance in coagulase-negative staphylococci isolated in blood cultures from patients with hematological malignancies during three decades. Eur J Clin Microbiol Infect Dis 30: 1349-1354.

Araujo GL, Coelho LR, Carvalho CB, Maciel RM, Coronado AZ, Rozenbaum R, Ferreira-Carvalho BT, Figueiredo AM, Teixeira LA 2006. Commensal isolates of methicillin-resistant Staphylococcus epidermidis are also well equipped to produce biofilm on polystyrene surfaces. J Antimicrob Chemother 57: 855-864.

Barros EM, Ceotto H, Bastos MC, dos Santos KR, Giambiagi-Demarval M 2012. Staphylococcus haemolyticus as an important hospital pathogen and carrier of methicillin resistance genes. J Clin Microbiol 50: 166-168.

Berglund C, Ito T, Ikeda M, Ma XX, Söderquist B, Hiramatsu K 2008. Novel type of staphylococcal cassette chromosome mec in a methicillin-resistant Staphylococcus aureus strain isolated in Sweden. Antimicrob Agents Chemother 52: 3512-3516.

Bouchami O, Hassen AB, de Lencastre H, Miragaia M 2012. High prevalence of mec complex $\mathrm{C}$ and $c c r C$ is independent of SCCmec type V in Staphylococcus haemolyticus. Eur J Clin Microbiol Infect Dis 31: 605-614.

Boye K, Bartels MD, Andersen IS, Moller JA, Westh H 2007. A new multiplex PCR for easy screening of methicillin-resistant Staphylococcus aureus SCCmec types I-V. Clin Microbiol Infect 13: 725-727.

CLSI - Clinical and Laboratory Standards Institute 2011. Performance standards for antimicrobial susceptibility testing, 19th Informational Supplement M100-S19, CLSI, Wayne, p. 52-59. de Almeida LM, Lincopan N, de Araújo MR, Mamizuka EM 2012. Clonal dissemination of linezolid-resistant Staphylococcus haemolyticus exhibiting the G2576T mutation in the 23S rRNA gene in a tertiary care hospital in Brazil. Antimicrob Agents Chemother 56: 2792-2793.

Fredheim EGA, Klingenberg C, Rohde H, Frankenberger S, Gaustad P, Flægstad T, Sollid JE 2009. Biofilm formation by Staphylococcus haemolyticus. J Clin Microbiol 47: 1172-1180.

Horan TC, Andrus M, Dudeck MA 2008. CDC/NHSN surveillance definition of health care-associated infection and criteria for specific types of infections in the acute care setting. Am J Infect Control 36: 309-332.

Keim LS, Torres-Filho SR, Silva PV, Teixeira LA 2011. Prevalence, aetiology and antibiotic resistance profiles of coagulase-negative Staphylococci isolated in a teaching hospital. Braz J Microbiol 42: 248-255.

Mombach PMAB, Reiter KC, Paiva RM, Barth AL 2007. Distribution of staphylococcal cassette chromosome mec (SCCmec) types I, II, III and IV in coagulase-negative staphylococci from patients attending a tertiary hospital in southern Brazil. J Med Microbiol 56: $1328-1333$.

Teixeira LA, Resende CA, Ormonde LR, Rosenbaum R, Figueiredo AMS, de Lencastre H, Tomasz A 1995. Geographic spread of epidemic multiresistant Staphylococcus aureus clone in Brazil. J Clin Microbiol 33: 2400-2404.

Zhang K, McClure JA, Elsayed S, Conly JM 2009. Novel staphylococcal cassette chromosome mec type, tentatively designated type VIII, harboring class A mec and type $4 \mathrm{ccr}$ gene complexes in a Canadian epidemic strain of methicillin-resistant Staphylococcus aureus. Antimicrob Agents Chemother 53: 531-540.

Zong Z, Peng C, Lü X 2011. Diversity of SCCmec elements in methicillin-resistant coagulase-negative staphylococci clinical isolates. PLoS ONE 6: e20191. 